\title{
The dependence of test-mass thermal noises on beam shape in gravitational-wave interferometers
}

\author{
Geoffrey Lovelace \\ Theoretical Astrophysics, California Institute of Technology, Pasadena, CA 91125, USA \\ E-mail: geoffrey@tapir.caltech.edu
}

Received 22 April 2007, in final form 27 July 2007

Published 21 August 2007

Online at stacks.iop.org/CQG/24/4491

\begin{abstract}
In second-generation, ground-based interferometric gravitational-wave detectors such as Advanced LIGO, the dominant noise at frequencies $f \sim$ $40 \mathrm{~Hz}$ to $\sim 200 \mathrm{~Hz}$ is expected to be due to thermal fluctuations in the mirrors' substrates and coatings which induce random fluctuations in the shape of the mirror face. The laser-light beam averages over these fluctuations; the larger the beam and the flatter its light-power distribution, the better the averaging and the lower the resulting thermal noise. In semi-infinite mirrors, scaling laws for the influence of beam shape on the four dominant types of thermal noise (coating Brownian, coating thermoelastic, substrate Brownian and substrate thermoelastic) have been suggested by various researchers and derived with varying degrees of rigour. Because these scaling laws are important tools for current research on optimizing the beam shape, it is important to firm up our understanding of them. This paper (1) gives a summary of the prior work and of gaps in the prior analyses, (2) gives a unified and rigorous derivation of all four scaling laws and (3) explores, relying on work by J Agresti, deviations from the scaling laws due to finite mirror size.
\end{abstract}

PACS number: $04.80 . \mathrm{Nn}$

\section{Introduction and summary}

Second-generation interferometric gravitational wave detectors such as Advanced LIGO will be approximately ten times more sensitive than the current LIGO interferometers, leading to an improvement in event rate such that the first few hours of data at design sensitivity will contain more signals than the entire year-long science run that is presently under way [1]. In advanced LIGO's most sensitive frequency band ( $f \sim 40$ to $200 \mathrm{~Hz}$ ), the sensitivity is limited by internal thermal noise, i.e, by noise in the substrates and reflective coatings of the mirrors (also called 'test masses') at the ends of the interferometer's arms (e.g., figure 1 of [2]). Lowering the internal thermal noise would increase advanced LIGO's event rate throughout that band. 
Internal thermal noise can be divided into two different types: Brownian thermal noise (due to imperfections in the substrate or coating material, which couple normal modes of vibration to each other) and thermoelastic noise (due to random flow of heat in the substrate or coating, which causes random thermal expansion). When the laser beam shape is Gaussian, the Brownian and thermoelastic noises in the substrate (e.g. [3]) and in the coating (e.g. [4, 5]) are well understood.

One way of lowering the internal thermal noise is to (i) flatten the shape of the laser beam that measures the test mass position so that it better averages over the mirror faces' fluctuating shapes, and (ii) enlarge it to the largest size permitted by diffraction losses. A specific enlarged, flattened shape, the mesa beam, has been proposed by O'Shaughnessy and Thorne and explored (theoretically) in detail by them, d'Ambrosio, Strigin and Vyatchanin [6-8] and by Agresti and DeSalvo [9, 10]. The mesa shape was found to reduce the thermal noise powers by factors of order 2, with corresponding significant increases in the distances to which the planned interferometers can search. Motivated by this, mesa beams are currently being explored experimentally $[11,12]$.

The mesa shape is unlikely to be optimal. Bondarescu and Chen $[13,14]$ and Pierro and collaborators [15] are currently seeking the optimal beam shape for each of the four types of noise; they are also seeking a balance between the competing demands of the four optimal shapes. Further research will require balancing practical aspects of mirror design against the (possibly impractical) ideal shapes.

In all this current research, a crucial tool is a set of scaling laws for the dependence of the four types of thermal noise on the beam shape, in the limit of a mirror that is large compared to the beam diameter (semi-infinite mirror). These scaling laws have been proposed by various researchers over the past several years, and they have been derived with varying degrees of rigour, and in some cases with unnecessarily restrictive assumptions. This prior work will be discussed and critiqued in section 2.2.

Because these scaling laws are so important for current research, this paper scrutinizes them and their accuracies in some detail. In section 2 the scaling laws and assumptions underlying them are presented and prior research on them is described. Then in section 3 a unified and rigorous derivation of all four scaling laws is presented. In section 4 the breakdown of the scaling laws due to finite mirror size is explored. Finally, in section 5 a few conclusions are given.

\section{The scaling laws and prior research on them}

\subsection{Model and summary}

To explore the effect of the beam shape on the internal thermal noise, I consider a cylindrical test mass substrate of radius $R$ and thickness $H$ and suppose that these size scales are comparable: $R \sim H$. I choose a cylindrical coordinate system $(r, \varphi, z)$ such that $r=0$ is the mirror axis, $z=0$ is the reflectively coated surface of the mirror substrate, and points with $0<z<H$ are inside the mirror substrate.

An axisymmetric laser beam with intensity profile $p(r)$ is normally incident on the mirror ${ }^{1}$. The intensity profile is normalized, so

$$
2 \pi \int_{0}^{R} \mathrm{~d} r r p(r)=1 .
$$

1 The shape of the mirror faces must also be changed slightly (by height changes $\lesssim$ one wavelength of the laser light) so that $p(r)$ is an eigenmode of the arm cavity. In this paper, I assume that the mirror faces take whatever shape is necessary to support a beam with intensity profile $p(r)$. 
The beam measures $q(t)$, a weighted average of the mirror's longitudinal position (equation (3) of $[16]^{2}$ ),

$$
q(t) \equiv \int_{0}^{2 \pi} \mathrm{d} \varphi \int_{0}^{R} \mathrm{~d} r r p(r) Z(r, \varphi, t) .
$$

Here $Z(r, \varphi, t)$ is the displacement (in the direction parallel to the laser beam) at time $t$ of a point at radius $r$ and angle $\varphi$ on the mirror surface.

In LIGO, so as to keep diffraction losses $\lesssim 1 \mathrm{ppm}$, the beam radius over which, say, 95\% of the signal $q(t)$ is collected, is kept significantly smaller than the mirror radius $R$ and thickness $H$. (In advanced LIGO, e.g., a typical fused-silica mirror has $R=17 \mathrm{~cm}$ and $H=20 \mathrm{~cm}$, while $95 \%$ of the signal is collected over a radius of $2 w \approx 0.6 R$ (figure 5 ) when the beam shape is Gaussian.) This motivates the idealization of the mirror as a semi-infinite slab bounded by a plane, $R \rightarrow \infty, H \rightarrow \infty$. (The accuracy of this infinite-test-mass (ITM) approximation will be discussed in section 4.2.)

Internal thermal noise will cause small fluctuations in $Z(r, \varphi, t)$. The (single-sided) spectral density $S_{q}$ associated with the measurement of the mirror position $q$ can be computed using Levin's thought experiment [16]:

(i) Deform the (semi-infinite) mirror with a force with amplitude $F$, frequency $f$ and pressure distribution $F p(r)$ the same as the light's intensity profile. Because the frequencies of interest (i.e. $f \sim 100 \mathrm{~Hz}$ ) are far below the lowest resonant frequencies of the mirror $f_{\text {res }} \sim 10^{4} \mathrm{~Hz}$, the hypothetical applied force can be idealized as static when computing the resulting strain of the mirror.

(ii) Compute the Brownian and thermoelastic dissipated power $W_{\text {diss }}$ due to the deformation caused by the applied force.

(iii) The fluctuation-dissipation theorem states that the dissipated power $W_{\text {diss }}$ is proportional to the spectral density $S_{q}$. Specifically, (equation (1) of [16])

$$
S_{q}(f)=\frac{2 k_{B} T}{\pi^{2} f^{2}} \frac{W_{\text {diss }}}{F^{2}} .
$$

Here $k_{B}$ is Boltzmann's constant and $T$ is the temperature of the material.

The interferometer does not directly measure the positions $q_{1,2,3,4}$ of the four test masses. If mirrors 1 and 2 are in one arm (of length $L=4 \mathrm{~km}$ ), and mirrors 3 and 4 are in the other arm (also of length $L$ ), then the interferometer actually measures the gravitational-wave strain $h \equiv\left[\left(q_{1}-q_{2}\right)-\left(q_{3}-q_{4}\right)\right] / L$. However, the spectral density $S_{h}$ corresponding to a measurement of $h$ can easily be obtained from $S_{q}$; because the noises in the four test masses are uncorrelated, the spectral density $S_{h}$ is just $S_{h}=\left(4 / L^{2}\right) S_{q}$. In the remainder of this paper, when referring to the noise of a single test mass, the subscript ' $q$ ' will be suppressed (i.e. $S \equiv S_{q}$ ), while the gravitational-wave-strain noise power will always be referred to as $S_{h}$.

In section 3.1, I compute the strain distribution that results from applying a force with amplitude $F$ and pressure distribution $F p(r)$ to a homogeneous, isotropic, semi-infinite mirror with a very thin reflective coating of a possibly different material. The calculation is a straightforward generalization of section 2 of [4]. In this calculation, I model the coating as a thin layer (of order microns, as compared to the $\mathrm{cm}$ size scales of the substrate) which adheres to the mirror surface. In section 3.2 I use the strain distributions to compute each of the four types of thermal noise $S(f)$. I find that if $p_{1}(r)$ and $p_{2}(r)$ are two different beam shapes, then

$$
\frac{S_{1, n}}{S_{2, n}}=\frac{\int_{0}^{\infty} \mathrm{d} k k^{n}\left[\tilde{p}_{1}(k)\right]^{2}}{\int_{0}^{\infty} \mathrm{d} k k^{n}\left[\tilde{p}_{2}(k)\right]^{2}},
$$

2 In the notation of [16], $q, p$ and $Z$ are called $x, f$ and $y$, respectively. 
where $n=1$ for coating Brownian and coating thermoelastic noise, $n=0$ for substrate Brownian noise and $n=2$ for substrate thermoelastic noise. Here $\tilde{p}(k)$ is (up to factors of $2 \pi)$ the two-dimensional Fourier transform of $p(r)$ over the surface of the mirror:

$$
\begin{aligned}
& \tilde{p}(k)=\int_{0}^{\infty} \mathrm{d} r r J_{0}(k r) p(r), \\
& p(r)=\int_{0}^{\infty} \mathrm{d} k k J_{0}(k r) \tilde{p}(k) .
\end{aligned}
$$

Here $J_{0}(x)$ is the 0th Bessel function of the first kind (the axisymmetry allows the 2D Fourier transform to reduce to a 1D Hankel transform).

If one knows $S_{1, n}$, computing $S_{2, n}$ amounts to computing simple integrals of $\tilde{p}(k)$. If one holds everything else fixed but changes the beam shape, the scaling law (4) makes it straightforward to determine the reduction of the thermal noises and the corresponding improvement in the interferometer sensitivity.

In the remainder of this paper, I derive these scaling laws, comment on their implications for advanced LIGO, and estimate their accuracy for finite test masses. In section 2.2, I discuss prior work related to the scaling laws. In section 3.1, I compute the deformation (characterized by the strain $S_{i j}$ ) due to a hypothetical applied force with amplitude $F$ and pressure distribution $F p(r)$. Then, in section 3.2, I compute the dissipated power $W_{\text {diss }}$ for the Brownian and thermoelastic dissipation in the coating and the substrate and insert $W_{\text {diss }}$ into equation (3) to determine how the noise depends on the beam shape. In section 4.1, I discuss implications of this result for advanced LIGO, and in section 4.2 I discuss the accuracies of the infinite-test-mass (ITM) scaling laws by comparing with others' finite-test-mass (FTM) predictions for the cases of Gaussian and mesa beam shapes. I make some concluding remarks in section 5.

\subsection{Discussion of prior research}

2.2.1. Thermoelastic substrate noise. In Levin's thought experiment, the dissipation associated with thermoelastic noise arises from heat flow down temperature gradients, which are induced by compression of the coating or substrate by an applied force. The increase in entropy corresponds to a dissipated power.

In 2003, in connection with his invention of the mesa beam and exploration of its properties, O'Shaughnessy used Levin's thought experiment to derive the following scaling law for the thermoelastic substrate noise:

$$
S_{\mathrm{sub}}^{\mathrm{TE}}(f)=c_{\mathrm{sub}}^{\mathrm{TE}}(f) \int_{0}^{\infty} \mathrm{d} k k^{2} \tilde{p}^{2}(k),
$$

where $c_{\mathrm{sub}}^{\mathrm{TE}}(f)$ does not depend on the beam shape. This scaling law ultimately motivated the other three. O'Shaughnessy included his derivation of this law as appendix $\mathrm{H}$ of his (as yet unpublished) 2004 paper with Strigin and Vyatchanin [8] on mesa beams. He used a slightly different (but no less rigorous) method from the unified derivation I give in section 3.2.4 (equation (39)). O'Shaughnessy wrote the scaling law in terms of 2D Fourier transforms; the reduction to 1D Hankel transforms makes numerical evaluation of the scaling law (6) very efficient (section 4.1).

2.2.2. Thermoelastic coating noise. Braginsky and Vyatchanin (appendix B.2 of [5]) and Fejer and collaborators (section IV D of [17]) have independently calculated the thermoelastic coating noise for Gaussian beam shapes (though the analysis in [5] is only valid when the 
coating and substrate elastic properties are identical [17]). Scrutinizing the derivation in [17], Thorne speculated in 2004 (unpublished) that the thermoelastic coating noise obeys a scaling law of the form

$$
S_{\text {coat }}^{\mathrm{TE}}(f)=c_{\text {coat }}^{\mathrm{TE}}(f) \int_{0}^{\infty} \mathrm{d} k k \tilde{p}^{2}(k) .
$$

In 2006 I verified Thorne's conjecture via almost trivial generalizations of the BraginskyVyatchanin and Fejer et al analyses; my derivation is given in section 3.2.2 (equation (29)). In 2006 O'Shaughnessy, learning of my work but not knowing my result, extended a clever dimensional analysis argument that he originally invented for Brownian coating noise (below) to the other three types of noises [18]; O'Shaughnessy's method is discussed in section 2.2.5.

2.2.3. Brownian coating noise. In Levin's thought experiment, the dissipation associated with Brownian thermal noise can be modelled as arising from a loss angle, which is an imaginary (i.e. damping) correction to the material's Young's modulus caused by coating or substrate imperfections.

In 2004, Thorne communicated to O'Shaughnessy and Vyatchanin his conjecture (7) for the scaling law for thermoelastic coating noise, and challenged them to find an analogous scaling law for Brownian coating noise. Independently, they each devised simple arguments that led to the law

$$
S_{\text {coat }}^{\mathrm{BR}}(f)=c_{\text {coat }}^{\mathrm{BR}}(f) \int_{0}^{\infty} \mathrm{d} r r p^{2}(r)=c_{\text {coat }}^{\mathrm{BR}}(f) \int_{0}^{\infty} \mathrm{d} k k \tilde{p}^{2}(k) .
$$

O'Shaughnessy [19] gave both an argument based on dimensional analysis (section 2.2.5) and a derivation for the special case that the substrate and coating have the same elastic properties. Vyatchanin's analysis [20] was based on a derivation for Gaussian beams, followed by an argument that if a beam with another shape $p(r)$ can be constructed by superposing Gaussian beams, then this scaling law must hold also for that other shape.

The scaling law (8) is local, i.e., the noise at a point on the mirror depends only on the beam intensity evaluated at that point. Thorne's intuition, however, led him to believe (incorrectly) that the scaling law should be nonlocal ${ }^{3}$. Consequently, Thorne was so highly sceptical of O'Shaughnessy's and Vyatchanin's arguments and the claimed scaling law that he-unfortunately_-dissuaded both O'Shaughnessy and Vyatchanin from publishing their arguments and result.

The following year (2005), Thorne, still sceptical of the O'Shaughnessy-Vyatchanin result (8), suggested to me that I carry out a detailed derivation of the Brownian-coating-noise scaling law from first principles. My analysis, based on Levin's method and reported in this paper, gave the result (8), in agreement with O'Shaughnessy and Vyatchanin, and motivated O’Shaughnessy to publish [19] his dimensional-analysis argument.

O'Shaughnessy's derivation is restricted (unrealistically) to identical elastic properties for substrate and coating. My derivation (equation (24) below) allows the substrate and the coating to have different elastic properties. Vyatchanin's derivation is valid only for those beam shapes that can be achieved by superposing Gaussians - though it might well be that any shape can be achieved in this way. My derivation is definitely valid for any axially symmetric beam shape $p(r)$.

\footnotetext{
3 It turns out (section 3.2.1) that nonlocal terms do appear at intermediate steps in the derivation but do not contribute to the scaling law itself.
} 
2.2.4. Brownian substrate noise. In 2005 Vinet proposed [21] the following scaling law for the substrate Brownian noise:

$$
S_{\mathrm{sub}}^{\mathrm{BR}}(f)=c_{\mathrm{sub}}^{\mathrm{BR}}(f) \int_{0}^{\infty} \mathrm{d} k \tilde{p}^{2}(k) .
$$

He deduced this law as a trivial consequence of his equations (1)-(3). He did not present a derivation of those equations, but he recognized that they can be obtained by generalizing the derivation in [22], which assumes that the beam shape is Gaussian. In section 3.2.3, I explicitly derive equation (9). In parallel with my work, O'Shaughnessy applied his dimensional analysis technique to verify Vinet's scaling law (9).

2.2.5. Dimensional analysis. O'Shaughnessy's dimensional analysis argument, referred to above, consists of three steps:

(i) The scaling laws must take the form of a translation-invariant inner product of $p(r)$ with itself, since the mirror is taken to be semi-infinite. In the Fourier domain, for axisymmetric beam shapes $p_{1}(r)$ and $p_{2}(r)$, the scaling law must then take the form

$$
\frac{S_{1}}{S_{2}}=\frac{\int_{0}^{\infty} \mathrm{d} k k \tilde{G}(k) \tilde{p}_{1}^{2}(k)}{\int_{0}^{\infty} \mathrm{d} k k \tilde{G}(k) \tilde{p}_{2}^{2}(k)} .
$$

(ii) The only length scale (other than the width of the beam) is the small coating thickness $d$, so $\tilde{G}(k)=k^{n} d$ for coating thermal noise and $\tilde{G}(k)=k^{n}$ for substrate thermal noise.

(iii) The power $n$ is chosen by demanding that when the beam shape is a Gaussian, the noise scales as the correct power of the beam width.

This argument turns out to produce the correct scaling laws, but, without sufficient care, it can also lead one amiss. For instance, when considering thermoelastic coating noise, step (ii) must be amended, since there is a second length scale: the characteristic length of diffusive heat flow [5, 17]. In his original manuscript [18], O'Shaughnessy neglected this second length scale, and incorrectly deduced that $n=3$ for coating thermoelastic noise. After I contacted O'Shaughnessy regarding this error, he corrected his analysis [19] and obtained the same result, $n=1$, as I had derived (section 3.2.2) below.

\section{Derivation of the infinite-test-mass (ITM) scaling laws}

\subsection{Strain of a semi-infinite body with thin facial coatings due to a static, axisymmetric force}

The thermal noise is determined by the symmetric part of the strain $S_{i j}$ that the test mass would experience if a normal force with pressure distribution $F p(r)$ were applied to the mirror surface. (Here and throughout this paper, the subscripts $i, j, k, \ldots$ label the components of vectors and tensors.) In this section, I evaluate $S_{i j}$ in the mirror substrate and coating. In section 3.2, I use these results to compute $W_{\text {diss }}$ (which, by equation (3), determines the thermal noise).

The spectral density $S$ is independent of $F$; therefore, for convenience I let $F=1$ throughout the rest of this paper. (The corresponding equations for generic values of $F$ may be obtained by letting $p(r) \rightarrow F p(r)$ and $\tilde{p}(k) \rightarrow F \tilde{p}(k)$. A factor of $F^{2}$ then appears in $W_{\text {diss }}$; the factor of $1 / F^{2}$ in equation (3) cancels it.)

If the displacement vector of an element at position $(r, \varphi, z)$ of the test mass is $u_{i}(r, z)$ (axisymmetry guarantees no explicit $\varphi$ dependence), then the strain $S_{i j}$ is $S_{i j} \equiv \nabla_{j} u_{i}$. In the orthonormal, cylindrical basis used here and throughout this paper, the components denoted by $i, j$ can take the values $r, \varphi, z$. Following the methods developed in [22] (but correcting 
some typographical errors), equation (19) of [3] gives the cylindrical components of the displacement of the test mass substrate:

$$
\begin{aligned}
& u_{r}(r, z)=\frac{1}{2 \mu} \int_{0}^{\infty} \mathrm{d} k J_{1}(k r) \mathrm{e}^{-k z}\left(1-\frac{\lambda+2 \mu}{\lambda+\mu}+k z\right) \tilde{p}(k), \\
& u_{\varphi}(r, z)=0 \\
& u_{z}(r, z)=\frac{1}{2 \mu} \int_{0}^{\infty} \mathrm{d} k J_{0}(k r) \mathrm{e}^{-k z}\left(1+\frac{\mu}{\lambda+\mu}+k z\right) \tilde{p}(k) .
\end{aligned}
$$

Here $\lambda$ and $\mu$ are the Lamé coefficients of the substrate, which are defined by the following relation between the substrate stress $T_{i j}$ and strain $S_{i j}$ :

$$
T_{i j}=-\lambda \theta \delta_{i j}-2 \mu S_{(i j)}
$$

Here $\theta \equiv S_{i i}$ is the expansion (i.e., the trace) of the strain. (Throughout this paper, the Einstein summation convention is used, so that repeated indices are summed over (i.e., $S_{i i} \equiv S_{r r}+S_{\varphi \varphi}+S_{z z}$ ). Also, here and throughout this paper, parentheses surrounding indices indicate that the symmetric part should be taken (i.e., $2 S_{(i j)} \equiv S_{i j}+S_{j i}$ ).) Note that the vector $u_{i}$ satisfies the equilibrium equation $\nabla_{j} T_{i j}=0$.

The components of the symmetric part of the strain are (with commas denoting partial derivatives)

$$
\begin{aligned}
& \theta=S_{i i} \equiv S_{r r}+S_{\varphi \varphi}+S_{z z}, \\
& S_{r r}=u_{r, r}=\theta-S_{z z}-S_{\varphi \varphi}, \\
& S_{\varphi \varphi}=\frac{u_{r}}{r}, \\
& S_{(r z)}=\frac{1}{2}\left(u_{r, z}+u_{z, r}\right), \\
& S_{z z}=u_{z, z}, \\
& S_{(r \varphi)}=S_{(\varphi z)}=0 .
\end{aligned}
$$

Evaluating the derivatives of equations $(11 a)-(11 c)$ and inserting the result into equations $(13 a)-(13 e)$ gives

$$
\begin{aligned}
& \theta=\frac{1}{2 \mu} \int_{0}^{\infty} \mathrm{d} k k J_{0}(k r)\left(\frac{-2 \mu}{\lambda+\mu}\right) \mathrm{e}^{-k z} \tilde{p}(k), \\
& S_{r r}=\theta-S_{z z}-S_{\varphi \varphi}, \\
& S_{\varphi \varphi}=\frac{1}{2 \mu} \int_{0}^{\infty} \mathrm{d} k \frac{J_{1}(k r)}{r} \mathrm{e}^{-k z}\left(1-\frac{\lambda+2 \mu}{\lambda+\mu}+k z\right) \tilde{p}(k), \\
& S_{(z r)}=-\frac{1}{2 \mu} \int_{0}^{\infty} \mathrm{d} k k J_{1}(k r)(k z) \mathrm{e}^{-k z} \tilde{p}(k), \\
& S_{z z}=\frac{1}{2 \mu} \int_{0}^{\infty} \mathrm{d} k k J_{0}(k r)\left(-\frac{\mu}{\lambda+\mu}-k z\right) \mathrm{e}^{-k z} \tilde{p}(k) .
\end{aligned}
$$

Setting $z=0$ in equations $(14 a)-(14 e)$ and combining with equation (5) yields the nonvanishing stresses on the substrate surface: 


$$
\begin{aligned}
\left.\theta\right|_{z=0} & =\left(\frac{-1}{\lambda+\mu}\right) p(r), \\
\left.S_{r r}\right|_{z=0} & =\frac{1}{2}\left(\frac{-1}{\lambda+\mu}\right) p(r)-\left.S_{\varphi \varphi}\right|_{z=0}, \\
\left.S_{\varphi \varphi}\right|_{z=0} & =\frac{1}{2}\left(\frac{-1}{\lambda+\mu}\right) \int_{0}^{\infty} \mathrm{d} k \frac{J_{1}(k r)}{r} \tilde{p}(k), \\
\left.S_{z z}\right|_{z=0} & =\frac{1}{2}\left(\frac{-1}{\lambda+\mu}\right) p(r) .
\end{aligned}
$$

Here I have used the identity

$$
\int_{0}^{\infty} \mathrm{d} k k J_{0}(k r) J_{0}\left(k r^{\prime}\right)=\frac{\delta\left(r^{\prime}-r\right)}{r^{\prime}} .
$$

Note that on the surface of the substrate $\theta$ and $S_{z z}$ are local (i.e., their values at any point depend only on the value of $p(r)$ at that point), while $S_{\varphi \varphi}$ is nonlocal. The component $S_{r r}$ can be written as the sum of a local part and a nonlocal part; the nonlocal part of $S_{r r}$ is just $-S_{\varphi \varphi}$.

The thin coating approximation gives the nonvanishing components of the coating strain in terms of the strain on the substrate surface (equation (A4) of [4]):

$$
\begin{aligned}
\theta^{\text {coat }} & =\left.\frac{\lambda+2 \mu_{\text {coat }}}{\lambda_{\text {coat }}+2 \mu_{\text {coat }}}\left(\theta-S_{z z}\right)\right|_{z=0}+\left.\frac{\lambda+2 \mu}{\lambda_{\text {coat }}+2 \mu_{\text {coat }}} S_{z z}\right|_{z=0}, \\
S_{r r}^{\text {coat }} & =\left.S_{r r}\right|_{z=0}=\theta^{\text {coat }}-S_{\varphi \varphi}^{\text {coat }}-S_{z z}^{\text {coat }}, \\
S_{\varphi \varphi}^{\text {coat }} & =\left.S_{\varphi \varphi}\right|_{z=0}, \\
S_{z z}^{\text {coat }} & =\left.\frac{\lambda-\lambda_{\text {coat }}}{\lambda_{\text {coat }}+2 \mu_{\text {coat }}}\left(\theta-S_{z z}\right)\right|_{z=0}+\left.\frac{\lambda+2 \mu}{\lambda_{\text {coat }}+2 \mu_{\text {coat }}} S_{z z}\right|_{z=0} .
\end{aligned}
$$

In [4], these conditions are said to hold in the limit that the Poisson ratio of the substrate and coating are 'not too different,' but this restriction is unnecessary (see appendix B).

Finally, after inserting equations $(15 a)-(15 d)$ into equations $(17 a)-(17 d)$ I conclude that $\theta^{\text {coat }}$ and $S_{z z}^{\text {coat }}$ are local; while $S_{\varphi \varphi}^{\text {coat }}$ and $S_{r r}^{\text {coat }}$ are nonlocal. However, this nonlocality turns out not to influence the coating noises. This is because, after using equation $(17 b)$ to eliminate $S_{r r}^{\text {coat }}$, it turns out that the remaining nonlocal part $S_{\varphi \varphi}^{\text {coat }}$ only appears in the coating $W_{\text {diss }}$ (according to equations (23) and (28)) via the integral

$$
\begin{aligned}
\int_{0}^{\infty} \mathrm{d} r r S_{(i j)}^{\text {coat }} S_{(i j)}^{\text {coat }} & =\int_{0}^{\infty} \mathrm{d} r r\left[\left(S_{r r}^{\text {coat }}\right)^{2}+\left(S_{\varphi \varphi}^{\text {coat }}\right)^{2}+\left(S_{z z}^{\text {coat }}\right)^{2}\right] \\
& =\int_{0}^{\infty} \mathrm{d} r r\left[\left(\theta^{\text {coat }}-S_{z z}^{\text {coat }}\right)^{2}+\left(S_{z z}^{\text {coat }}\right)^{2}+2\left(S_{\varphi \varphi}^{\text {coat }}\right)^{2}-2 S_{\varphi \varphi}^{\text {coat }}\left(\theta^{\text {coat }}-S_{z z}^{\text {coat }}\right)\right] .
\end{aligned}
$$

In appendix A, I show that

$$
\int_{0}^{\infty} \mathrm{d} r r\left(S_{\varphi \varphi}^{\text {coat }}\right)^{2}-S_{\varphi \varphi}^{\text {coat }}\left(\theta^{\text {coat }}-S_{z z}^{\text {coat }}\right)=0,
$$

so only the local parts of the strain $\left(\theta^{\text {coat }}\right.$ and $\left.S_{z z}^{\text {coat }}\right)$ influence the thermal noise. This fact turns out to imply local coating scaling laws in agreement with O'Shaughnessy's [19] and Vyatchanin's [20] arguments (section 3.2). 


\subsection{Internal thermal noise}

3.2.1. Brownian coating noise. For Brownian thermal noise in an elastic material, the dissipated power is (equation (12) of [16] with a static applied force and with $U=$ $\left.-(1 / 2) S_{i j} T_{i j}\right)$

$$
W_{\mathrm{diss}}=-\pi f \int_{0}^{d} \mathrm{~d} z \int_{0}^{2 \pi} \mathrm{d} \varphi \int_{0}^{\infty} \mathrm{d} r r \phi(f) S_{i j} T_{i j} .
$$

Here $\phi$ is the loss angle (i.e., the imaginary, damping part of the Young's modulus of the coating material) and $T_{i j}$ is the stress. When the material is the thin reflective coating of a mirror, there are effectively two loss angles [4], $\phi_{\|}$and $\phi_{\perp}$, defined so that in the previous equation

$$
\begin{aligned}
\phi(f) S_{i j} T_{i j} & \rightarrow \phi_{\|}(f)\left(S_{r r}^{\text {coat }} T_{r r}^{\text {coat }}+S_{\varphi \varphi}^{\text {coat }} T_{\varphi \varphi}^{\text {coat }}\right)+\phi_{\perp}(f) S_{z z}^{\text {coat }} T_{z z}^{\text {coat }} \\
& =\phi_{\|}(f) S_{i j}^{\text {coat }} T_{i j}^{\text {coat }}+\left(\phi_{\perp}-\phi_{\|}\right) S_{z z}^{\text {coat }} T_{z z}^{\text {coat }} .
\end{aligned}
$$

This result can be obtained by combining equations (4) and (13)-(15) of [4] with equation (9) of [16] and recalling that in the coating, the strain (equations $(17 a)-(17 d)$ ) is diagonal.

For a homogeneous coating, the stress $T_{i j}^{\text {coat }}$ is

$$
T_{i j}^{\text {coat }}=-\lambda^{\text {coat }} \theta^{\text {coat }} \delta_{i j}-2 \mu^{\text {coat }} S_{(i j)}^{\text {coat }},
$$

where $\lambda^{\text {coat }}$ and $\mu^{\text {coat }}$ are the Lamé coefficients of the coating, $S_{(i j)}^{\text {coat }}$ is the symmetric part of the coating strain and $\theta \equiv S_{i i}$ is the expansion. Combining equations (21), (20) and (22) gives the following expression for $W_{\text {diss }}$ :

$W_{\mathrm{diss}}=2 \pi^{2} f d \phi_{\|}(f) \int_{0}^{\infty} \mathrm{d} r r A+2 \pi^{2} f d\left[\phi_{\perp}(f)-\phi_{\|}(f)\right] \int_{0}^{\infty} \mathrm{d} r r B$,

$A=\left(\lambda^{\text {coat }} \theta_{\text {coat }}^{2}+2 \mu^{\text {coat }} S_{(i j)}^{\text {coat }} S_{(i j)}^{\text {coat }}\right)$,

$B=S_{z z}^{\text {coat }}\left(\lambda^{\text {coat }} \theta^{\text {coat }}+2 \mu^{\text {coat }} S_{z z}^{\text {coat }}\right)$.

Combining equations (23), (17a)-(17d), (15a)-(15d) and (19) and then inserting the result into equation (3) gives the spectral density $S$ of the Brownian coating noise. However, for the present purpose, only terms involving the beam shape are relevant. Absorbing all other terms into a single term $c_{\text {coat }}^{\mathrm{BR}}(f)$ yields

$$
S_{\text {coat }}^{\mathrm{BR}}(f)=c_{\text {coat }}^{\mathrm{BR}}(f) \int_{0}^{\infty} \mathrm{d} r r p^{2}(r) .
$$

This is a local scaling law; i.e., the noise at each point on the mirror's surface is proportional to the square of the beam intensity there. This law is the same as O'Shaughnessy's [19] and Vyatchanin's [20] scaling law for the Brownian coating thermal noise.

Parseval's equation (which follows from equation (5)) makes it easy to rewrite this scaling law in the Fourier domain, which will facilitate comparison with the substrate noise. The result is

$$
S_{\text {coat }}^{\mathrm{BR}}(f)=c_{\text {coat }}^{\mathrm{BR}}(f) \int_{0}^{\infty} \mathrm{d} k k \tilde{p}^{2}(k)
$$

3.2.2. Thermoelastic coating noise. The calculation of the thermoelastic coating noise is similar to the calculation of Brownian coating noise. But now, in response to the static, normal 
applied pressure, the dissipated power $W_{\text {diss }}$ is caused by heat flow, $\propto \nabla \delta T$, down a temperature gradient $\nabla \delta T$ caused by the material's deformation:

$$
W_{\text {diss }}=\frac{\pi \kappa}{T} \int_{0}^{\infty} \mathrm{d} z \int_{0}^{\infty} \mathrm{d} r r(\nabla \delta T)^{2}
$$

(equation (5) of [3] in the case of a static applied force and after evaluating the time average and trivial $\varphi$ integral). Here $T$ is the temperature of the coating in the absence of the deformation and $\kappa$ is the material's coefficient of thermal conductivity.

Braginsky and Vyatchanin [5] and Fejer and collaborators [17] have independently solved for the thermoelastic coating noise. The results obtained in [5] are correct only when the coating and substrate have the same elastic properties (section I in [17]); however, this restriction is not relevant here, since [17] and [5] agree on the coating thermoelastic noise's dependence on the beam shape $p(r)$.

If the temperature change were adiabatic, $\delta T$ would simply be proportional to $\theta^{\text {coat }}$ (see, e.g., equation (12) of [3]). (Physically, this simply means that the temperature of an element in the coating changes linearly with volume.) However, as noted in [5], for frequencies of interest $\left(f \sim 100 \mathrm{~Hz}\right.$ ), the diffusive heat characteristic length $\ell_{D}(f)$ of the substrate and coating (of the order of $\mathrm{mm}$ ) is far larger than the coating thickness $d$ (which is of the order of a few microns). Because diffusive heat flow in the longitudinal direction is not negligible, heat flow in the direction normal to the coating cannot be treated adiabatically [5]. By contrast, the substrate thermoelastic noise can be treated adiabatically (section 3.2.4), as can the heat flow in the plane of the coating ('tangential' heat flow).

Because the tangential heat flow is adiabatic, $\partial \delta T / \partial r \propto \theta / w$, where $w \sim \mathrm{cm}$ is the length scale over which $p(r)$ varies. On the other hand, $\partial \delta T / \partial z \propto \theta / \ell_{D}$, where $\ell_{D} \sim \mathrm{mm}$ is the diffusive heat characteristic length. Because the tangential derivatives are much smaller than the longitudinal derivatives, all derivatives except $\partial / \partial z$ may be neglected. It follows that $W_{\text {diss }}$ will depend only on $p(r)$ and not on its radial derivatives.

Based on these observations, Braginsky and Vyatchanin [5] and Fejer and collaborators [17] solve the thermoconductivity equation (e.g., equation (1) of [17]) for the temperature perturbations $\delta T$. Both [5] and [17] assume that the beam shape is Gaussian, but it is quite easy to generalize their arguments to non-Gaussian beam shapes. Combining equations (B5)(B7), (66) and (68) of [17] (but now regarding their function $\rho(r)$ as a generic beam shape) shows that the temperature perturbations in the coating have the form

$$
\delta T \propto p(r) \times F(z),
$$

where $F(z)$ is a function of $z$ only. (Equivalently, equation (27) can be obtained by combining equations (B.10) and (B.12) of [5] (but now regarding $\theta$ as an expansion corresponding to a generic beam shape) with equations $(15 a)$ and $(17 a)$.) The precise form of $F(z)$ is given in [5] and [17] but is not needed in the present discussion.

Next, Braginsky and Vyatchanin compute the squared gradient $(\nabla \delta T)^{2} \simeq(\partial \delta T / \partial z)^{2}$ in equation (26) to obtain $W_{\text {diss }}$; Fejer and collaborators instead compute $W_{\text {diss }}$ by considering the interaction of (i) the unperturbed stress and strain (i.e., the stress and strain due to $p(r)$ when temperature perturbations are neglected), and (ii) the (complex) perturbations of the stress and strain caused by the small temperature perturbations $\delta T$. Both methods lead to the following expression for $W_{\text {diss }}$ : (equations (B.13) and (B.10) of [5]; equation (69) of [17])

$$
W_{\text {diss }}=\text { const } \times \int_{0}^{\infty} \mathrm{d} r r p^{2}(r) .
$$

Plugging this result into equation (3) gives the scaling law

$$
S_{\text {coat }}^{\mathrm{TE}}(f)=c_{\text {coat }}^{\mathrm{TE}}(f) \int_{0}^{\infty} \mathrm{d} r r p^{2}(r)=c_{\text {coat }}^{\mathrm{TE}}(f) \int_{0}^{\infty} \mathrm{d} k k \tilde{p}^{2}(k) .
$$


This is the same scaling law as for Brownian coating thermal noise. The coating thermoelastic noise is local and is the same as O'Shaughnessy's [19] and Vyatchanin's [20] law for Brownian coating thermal noise.

3.2.3. Brownian substrate noise. For Brownian substrate thermal noise there is only one relevant loss angle, $\phi$, so the dissipated power is (equation (49) of [3] with a static applied force)

$$
W_{\text {diss }}=2 \pi^{2} f \phi(f) \int_{0}^{\infty} \mathrm{d} z \int_{0}^{\infty} \mathrm{d} r r\left(\lambda \theta^{2}+2 \mu S_{(i j)} S_{(i j)}\right) .
$$

The integral of the squared strain can be expanded as

$$
\begin{aligned}
& \int_{0}^{\infty} \mathrm{d} z \int_{0}^{\infty} \mathrm{d} r r S_{(i j)} S_{(i j)}=\int_{0}^{\infty} \mathrm{d} z \int_{0}^{\infty} \mathrm{d} r r\left(S_{r r}^{2}+S_{\varphi \varphi}^{2}+S_{z z}^{2}\right) \\
&=\int_{0}^{\infty} \mathrm{d} z \int_{0}^{\infty} \mathrm{d} r r\left[\left(\theta-S_{z z}\right)^{2}+S_{z z}^{2}+2 S_{(r z)}^{2}+2 S_{\varphi \varphi}^{2}-2 S_{\varphi \varphi}\left(\theta-S_{z z}\right)\right] .
\end{aligned}
$$

In appendix $\mathrm{A}$, I show that

$$
\int_{0}^{\infty} \mathrm{d} r r\left[S_{\varphi \varphi}^{2}-S_{\varphi \varphi}\left(\theta-S_{z z}\right)\right]=0 .
$$

Substituting this result into equation (31) yields

$$
W_{\text {diss }}=2 \pi^{2} f \phi(f) \int_{0}^{\infty} \mathrm{d} z \int_{0}^{\infty} \mathrm{d} r r\left[\lambda \theta^{2}+2 \mu\left(\theta-S_{z z}\right)^{2}+2 \mu S_{z z}^{2}+4 \mu S_{(r z)}^{2}\right] \text {. }
$$

This expression can be evaluated term by term. Inserting equation (14a) into the integral of $\theta^{2}$ gives

$$
\begin{aligned}
I_{\theta} & \equiv \int_{0}^{\infty} \mathrm{d} z \int_{0}^{\infty} \mathrm{d} r r \theta^{2} \\
& =\frac{1}{4 \mu^{2}}\left(\frac{2 \mu}{\lambda+\mu}\right)^{2} \int_{0}^{\infty} \mathrm{d} k k \tilde{p}(k) \int_{0}^{\infty} \mathrm{d} k^{\prime} k^{\prime} \tilde{p}\left(k^{\prime}\right) \int_{0}^{\infty} \mathrm{d} z \mathrm{e}^{-\left(k+k^{\prime}\right) z} \int_{0}^{\infty} \mathrm{d} r r J_{0}(k r) J_{0}\left(k^{\prime} r\right) .
\end{aligned}
$$

Using the identity

$$
\int_{0}^{\infty} \mathrm{d} r r J_{n}(k r) J_{n}\left(k^{\prime} r\right)=\frac{\delta\left(k-k^{\prime}\right)}{k}
$$

on equation (34) and evaluating the integral over $z$ yields

$$
I_{\theta}=\frac{1}{8 \mu^{2}}\left(\frac{2 \mu}{\lambda+\mu}\right)^{2} \int_{0}^{\infty} \mathrm{d} k \tilde{p}^{2}(k) .
$$

The other terms in equation (33) can be evaluated similarly; they all turn out to have the same dependence on $\tilde{p}(k)$ as $I_{\theta}$. Inserting this result for $W_{\text {diss }}$ into equation (3) gives the scaling law

$$
S_{\mathrm{sub}}^{\mathrm{BR}}(f)=c_{\mathrm{sub}}^{\mathrm{BR}}(f) \int_{0}^{\infty} \mathrm{d} k \tilde{p}^{2}(k) .
$$

This scaling law is the same as the scaling law (25) for the coating thermal noise except that the $z$ integration has reduced the power of $k$ in the integrand by one. This scaling law agrees with equations (1) and (2) of [21]. 
3.2.4. Thermoelastic substrate noise. In contrast to the case of coating thermoelastic noise, the substrate thermoelastic noise can be treated using the adiabatic approximation. Therefore, the temperature perturbations $\delta T$ that drive the substrate thermoelastic noise $S_{\text {sub }}^{\mathrm{TE}}$ are proportional to the expansion, i.e. $\delta T \propto \theta$. This implies (e.g., by equation (26), or equation (13) of [3])

$$
S_{\mathrm{sub}}^{\mathrm{TE}}(f)=c_{\mathrm{sub}}^{\mathrm{TE}}(f) \int_{0}^{\infty} \mathrm{d} z \int_{0}^{\infty} \mathrm{d} r r(\nabla \theta)^{2}
$$

with $c_{\text {sub }}^{\mathrm{TE}}(f)$ being independent of the strain (and thus also the beam shape). Inserting equation (14a) into equation (38) gives the scaling law; after absorbing all constants into $c_{\text {sub }}^{\mathrm{TE}}(f)$, it takes the form

$$
S_{\mathrm{sub}}^{\mathrm{TE}}(f)=c_{\mathrm{sub}}^{\mathrm{TE}}(f) \int_{0}^{\infty} \mathrm{d} k k^{2} \tilde{p}^{2}(k),
$$

which O'Shaughnessy, Strigin and Vyatchanin obtain in [8]. This scaling law is the same as the scaling law (37) for the substrate Brownian noise except that the gradient raises the power of $k$ by 2 .

\section{Applying the ITM scaling laws to second-generation gravitational-wave interferometers}

To illustrate the scaling laws (24), (29), (37) and (39), suppose that the noise $S_{\tau, k}$ (with beam shape $p_{k}(r)$ and thermal noise type $\tau$ ) is known. Here and throughout the remainder of this paper, $\tau$ is a label that takes one of the following values: coating Brownian (Coat BR), coating thermoelastic (Coat TE), substrate Brownian (Sub BR) or substrate thermoelastic (Sub TE).

Now, if the beam shape were changed to $p_{u}(r)$ (while holding everything ${ }^{4}$ else fixed), then the unknown noise $S_{\tau, u}$ (with beam shape $p_{u}(r)$ ) would be (equation (4))

$$
S_{\tau, u}=C_{\mathrm{ITM}}^{2}\left[\tau ; p_{u}, p_{k}\right] S_{\tau, k},
$$

with

$$
C_{\mathrm{ITM}}^{2}\left[\tau ; p_{u}, p_{k}\right] \equiv \frac{\int_{0}^{\infty} \mathrm{d} k k^{n(\tau)} \tilde{p}_{u}^{2}(k)}{\int_{0}^{\infty} \mathrm{d} k k^{n(\tau)} \tilde{p}_{k}^{2}(k)}
$$

and

$$
n(\tau) \equiv\left\{\begin{array}{lll}
1 & : & \tau=\text { Coat BR or Coat TE } \\
0 & : & \tau=\text { Sub BR } \\
2 & : & \tau=\text { Sub TE }
\end{array}\right.
$$

When the beam shape is changed from $p_{k}$ to $p_{u}$, the noise amplitude (i.e., $\sqrt{S}$ ) is scaled by a factor of $C_{\mathrm{ITM}}\left[\tau ; p_{u}, p_{k}\right]$. Note that the gravitational-wave event rate is inversely proportional to the cube of the noise amplitude (e.g., [1]).

\subsection{Implications for advanced LIGO}

In advanced LIGO, the thermal noise may be significantly reduced by changing the shape of the laser beam. One proposal is to replace the Gaussian beam shape with a mesa beam (also called a flat-top beam) [6]. O’Shaughnessy, Strigin and Vyatchanin [8] have calculated

\footnotetext{
4 Since here I am neglecting edge effects, 'everything' means the temperature, the materials' elastic and thermal properties, the coating thickness, and the frequency. In section 4.2, when edge effects are considered, it will be the diffraction loss, not the mirror size, that is held fixed.
} 
the resulting reduction in substrate thermoelastic noise, Vinet has done the same for substrate Brownian thermal noise [21] and Agresti [2] and Agresti and DeSalvo [9, 10] have done the same for both substrate and coating thermal noises - all for the realistic case of finite mirrors. The reduction in thermal noise can also be understood as a consequence of the simple ITM scaling laws derived above. Although I only compare Gaussian and flat-top beams here, the scaling law given in equation (4) makes it simple-if one neglects finite-test-mass (FTM) effects - to compute the relative change in noise for any two beam shapes.

The normalized Gaussian beam shape is

$$
p_{\text {gauss }}(w ; r)=\frac{\mathrm{e}^{-r^{2} / w^{2}}}{\pi w^{2}}
$$

where $w$ is the width of the Gaussian beam. It is straightforward to compute $\tilde{p}_{\text {gauss }}(w ; k)$, since the integral can be done analytically; the result is

$$
\tilde{p}_{\text {gauss }}(w ; k)=\int_{0}^{\infty} \mathrm{d} r r J_{0}(k r) \frac{\mathrm{e}^{-r^{2} / w^{2}}}{\pi w^{2}}=\frac{1}{2 \pi} \mathrm{e}^{-k^{2} w^{2} / 4} .
$$

In position space, the mesa beam can be written as (equation (2.5) of [7])

$p_{\text {mesa }}(D ; r)=N\left|2 \pi \int_{0}^{D} \mathrm{~d} r^{\prime} r^{\prime} \exp \left[-\frac{\left(r^{2}+r^{\prime 2}\right)(1-\mathrm{i})}{2 b^{2}}\right] \times I_{0}\left[\frac{r r^{\prime}(1-\mathrm{i})}{b^{2}}\right]\right|^{2}$.

Here $D$ is a measure of the width of the beam, $b \equiv \sqrt{\lambda L / 2 \pi}$, with $L=4 \mathrm{~km}$ being the arm length and $\lambda=1064 \mathrm{~nm}$ the wavelength of the laser beam's primary frequency, and $N$ is a normalization constant adjusted so equation (1) is satisfied. Note that $p_{\text {mesa }}(r)$ must be evaluated numerically; to compute $\tilde{p}(k)$ efficiently, I use the fast Hankel transform algorithm [23].

Examples of the Gaussian and mesa shapes are plotted in figure 1. In figure 2, the width parameters $w$ and $D$ of a sequence ${ }^{5}$ of Gaussian and mesa beams are plotted as a function of mirror radius $R$ for beams with $1 \mathrm{ppm}$ of diffraction loss in the clipping approximation ${ }^{6}$. The ratio $D / w$ is also shown on the bottom horizontal axis. It is sometimes useful to regard $D$ and $w$ (for $1 \mathrm{ppm}$ losses) as functions of $D / w$ rather than of $R$-with $D / w$ actually being a surrogate for $R$.

The following three cases use equations (40)-(42) to illustrate how the thermal noise in advanced LIGO changes with different choices of Gaussian and mesa beam shapes.

4.1.1. Noise of a resized Gaussian beam. Suppose $p_{k}(r)=p_{\text {gauss }}\left(w_{o} ; r\right)$. Then the thermal noises for a Gaussian beam of some different size $w$ are determined by evaluating $C_{\text {ITM }}\left[\tau ; p_{\text {gauss }}(w ; r), p_{\text {gauss }}\left(w_{o} ; r\right)\right]$ (equation (41)) and inserting the result into equation (40). In this well-known case (see, e.g., the discussion and references in [10]), $C_{\text {ITM }}$ can be evaluated analytically, yielding the following relation:

$$
C_{\text {ITM }}^{2}\left[\tau ; p_{\text {gauss }}(w ; r), p_{\text {gauss }}\left(w_{o} ; r\right)\right] \propto \frac{1}{w^{n(\tau)+1}} .
$$

In the left panel of figure $3, C_{\mathrm{ITM}}\left[\tau ; p_{\text {gauss }}(w ; r), p_{\text {gauss }}\left(w_{o} ; r\right)\right]$ is plotted as a function of the beam width $w$.

5 This particular sequence was chosen to facilitate comparison with the results of [10], which includes edge effects. 6 In the clipping approximation, the diffraction loss is simply $2 \pi \int_{R}^{\infty} \mathrm{d} r r p(r)$, where $R$ is the mirror radius. In the ITM approximation, $R$ is larger than all other length scales; however, the actual, finite value of $R$ must be used in the clipping approximation for the diffraction loss to be nonvanishing. 


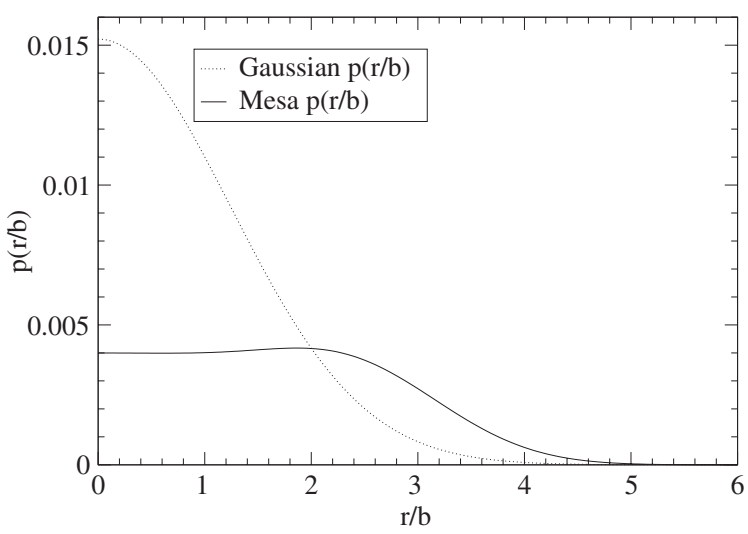

Figure 1. A plot of $p_{\text {gauss }}(r / b)$ and $p_{\text {mesa }}(r / b)$ for beams with 1 ppm diffraction losses (in the clipping approximation) on a mirror with radius $R=17 \mathrm{~cm}$. Here $b=\sqrt{L \lambda / 2 \pi}=2.6 \mathrm{~cm}$ is the width of the smallest Gaussian beam that can resonate in a LIGO arm cavity with length $L=4 \mathrm{~km}$ and light wavelength $\lambda=1064 \mathrm{~nm}$.

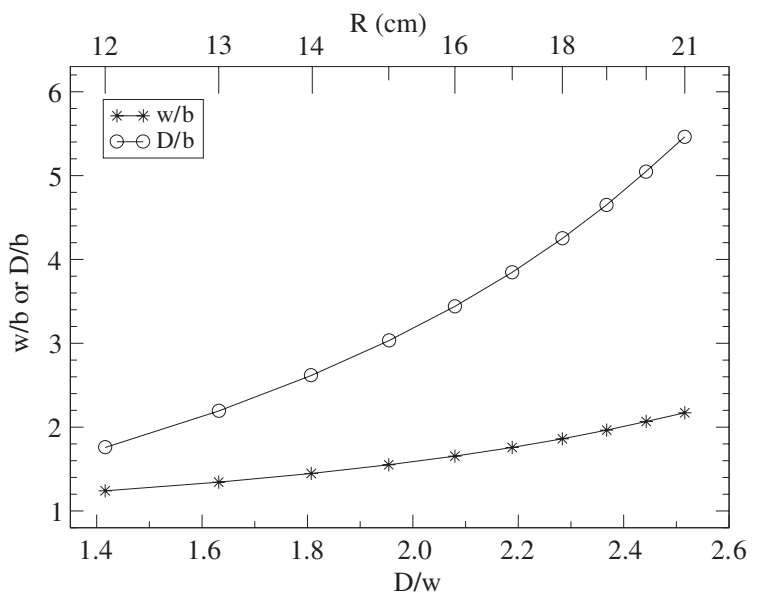

Figure 2. A plot of the Gaussian beam-width parameter $w$ and mesa beam-width parameter $D$ as a function of mirror radius $R$ (top of figure), for mirrors with $1 \mathrm{ppm}$ diffraction loss in the clipping approximation. The ratio $D / w$ is shown on the bottom of the figure. The parameter $b$ is defined in figure 1 .

4.1.2. Noise of a resized mesa beam. Suppose $p_{k}(r)=p_{\text {mesa }}\left(D_{o} ; r\right)$. Then the thermal noises for a mesa beam of some different size $D$ are determined by evaluating $C_{\text {ITM }}\left[\tau ; p_{\text {mesa }}(D ; r), p_{\text {mesa }}\left(D_{o} ; r\right)\right]$ (equation (41)) and inserting the results into equation (40). As shown in the right panel of figure 3 , in this case $C_{\text {ITM }}$ does not scale as an exact power of $D$ (although the actual relations are very well approximated by power laws).

4.1.3. Noise reduction by switching from a Gaussian beam to a mesa beam with the same diffraction loss and mirror radius. Finally, the scaling law (40) can be used to estimate the reduction in thermal noise by switching from a Gaussian beam to a mesa beam that has the same clipping-approximation diffraction loss on a mirror of the same radius. 

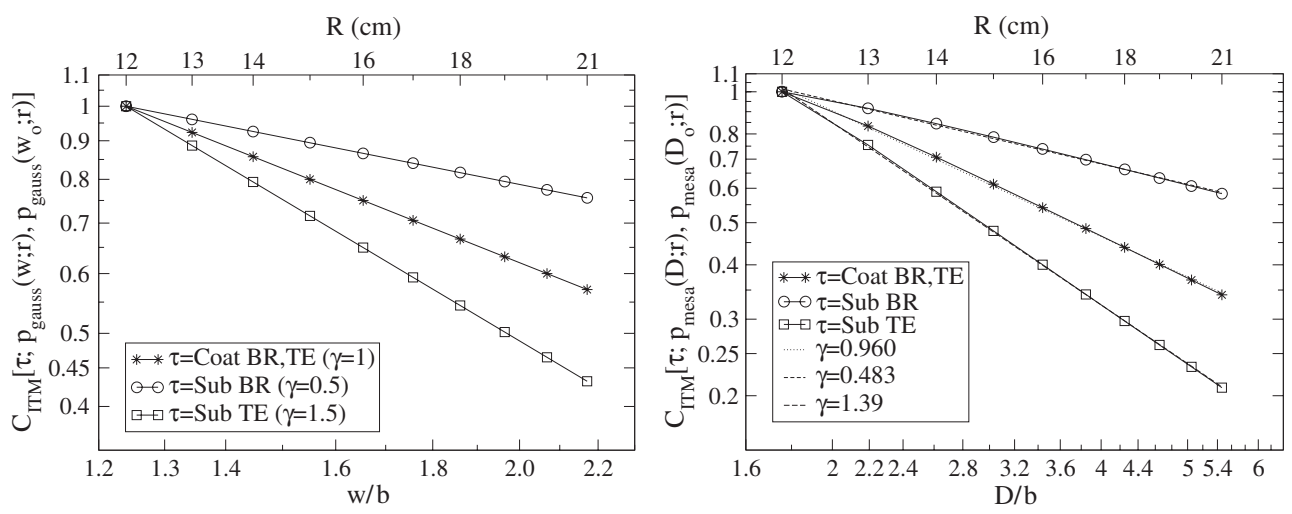

Figure 3. The scaling of thermal noises with Gaussian beam width $w$ (left panel) and mesa beam width parameter $D$ (right panel) in the infinite-test-mass (ITM) approximation. More specifically, $\log -\log$ plots of $C_{\text {ITM }}\left[\tau ; p_{\text {gauss }}(w ; r), p_{\text {gauss }}\left(w_{o} ; r\right)\right]$ and $C_{\text {ITM }}\left[\tau ; p_{\text {mesa }}(D ; r), p_{\text {mesa }}\left(D_{o} ; r\right)\right]$ as functions of $w / b$ and $D / b$, respectively. Here $w_{o} / b=1.24$ and $D_{o} / b=1.76$, both of which correspond to a mirror radius $R=12 \mathrm{~cm}$ and $1 \mathrm{ppm}$ diffraction losses. The Gaussian-beam noises exactly satisfy power laws of the form $C \propto 1 / w^{\gamma}$, and the mesa-beam noises are well approximated by power laws of the form $C \propto 1 / D^{\gamma}$.

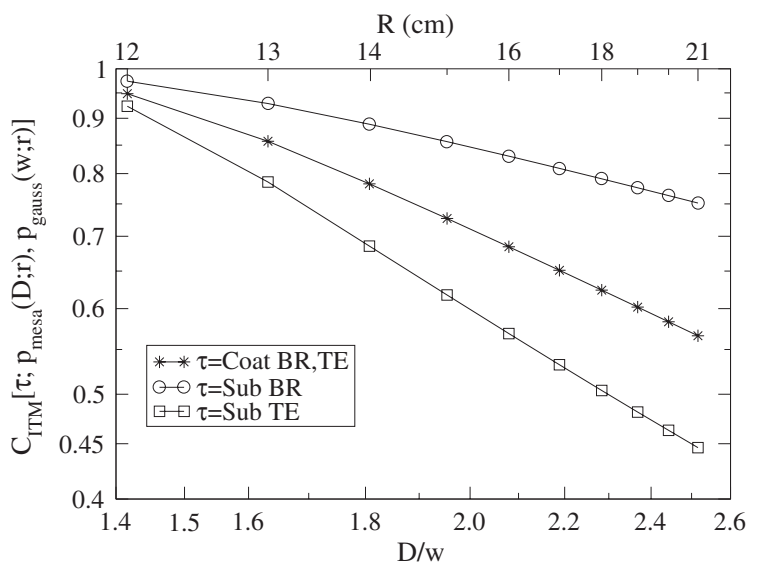

Figure 4. The reduction of thermal noise when mesa beams are used instead of Gaussian beams. More specifically, a $\log -\log$ plot of $C_{\mathrm{ITM}}\left[\tau ; p_{\text {mesa }}(D ; r), p_{\text {gauss }}(w ; r)\right]$ as a function of $D / w$. For each mirror radius $R, w$ and $D$ are chosen so that the diffraction losses are $1 \mathrm{ppm}$ in the clipping approximation.

Two complications in the resized-beam scalings are not present when scaling from Gaussian to mesa beams. First, while the original and resized beams were associated with different-sized mirrors, now the Gaussian and mesa beams are associated with the same mirror. Second, when relating the Gaussian and mesa beams, there is no need to specify a fiducial beam size (i.e. there is no analogue of $w_{o}$ and $D_{o}$ ). Without these two complications, the Gaussian-to-mesa scaling is perhaps conceptually cleaner than the resized-beam scalings.

Figure 4 shows $C_{\text {ITM }}\left[\tau ; p_{\text {mesa }}(D ; r), p_{\text {gauss }}(w ; r)\right]$ for the sequence of beams shown in figure 2 (beams with 1 ppm diffraction loss in mirrors of the same radius $R$ ). The thermal noise monotonically decreases with the mirror radius $R$, or equivalently, with $D / w$; however, when 
edge effects (i.e. finite-test-mass effects) are included, there is a limit to how much the noise can be reduced (section 4.2).

Neglecting edge effects, the ITM scaling laws can be used to estimate how much the noise can be reduced by switching from mesa beams to Gaussian beams. In advanced LIGO's most sensitive frequency band $(\sim 40 \mathrm{~Hz}$ to $\sim 200 \mathrm{~Hz}$ ), the total noise is dominated by the thermal noise of the mirrors; for mirrors made of fused silica (the baseline material for advanced LIGO [1]), the thermal noise is dominated by Brownian coating noise (e.g., [9]). Therefore, the coating-noise ITM scaling law can be used to estimate the dependence of the total noise (and thus event rates) on beam shape for sources in advanced LIGO's most sensitive frequency band (such as binary-neutron-star inspirals (e.g., figures 2 and 3 of [24])).

For a mirror radius of $17 \mathrm{~cm}$ (the baseline radius for advanced LIGO [1]), the coating-noise ITM scaling law predicts that the Brownian coating noise is reduced by a factor of $C_{\text {ITM }} \approx 0.65$ (figure 4) when the beam shape is changed from Gaussian to mesa (with everything else held fixed). Consequently, the rate at which advanced LIGO should detect binary-neutron-star inspirals would be a factor of about $1 / C_{\mathrm{ITM}}^{3} \approx 3.6$ higher when a mesa beam is used, as compared to the Gaussian-beam rate.

For mirrors made of sapphire (formerly considered for use in advanced LIGO), the noise is dominated by thermoelastic substrate noise (e.g., [8]), which (figure 4) is reduced by a larger factor than are the coating noises. For the same mirror size of $17 \mathrm{~cm}$, the ITM scaling laws predict that the thermoelastic substrate noise is reduced by a factor of $C_{\text {ITM }} \approx 0.532$.

This apparent advantage over fused silica disappears when edge effects are considered; however, it turns out that for the mirror sizes considered in this paper, the ITM approximation is much worse for estimating substrate noises than for coating noises (figure 8). When edge effects are taken into account [9], switching from Gaussian to mesa beams leads to a greater noise reduction for fused silica than for sapphire.

\subsection{Errors due to neglecting finite-test-mass (FTM) effects}

In the previous section, the ITM scaling laws predicted that if the diffraction losses are held fixed, then the coating and substrate noises decrease monotonically with increasing beam width (figures 3 and 4). In other words, for a given diffraction loss, the optimal beam width is simply 'as large as possible'.

However, this conclusion is only as strong as the ITM approximation. Its validity can be checked by comparing the beam widths to the corresponding mirror dimensions. In our modelling, the mirror radii $R$ are adjusted to maintain a constant clipping-approximation diffraction loss (CADL), while the thicknesses $H$ is then determined by requiring the mirror mass be $40 \mathrm{~kg}$ - the design specification for Advanced LIGO. (Thus $H$ will depend on whether the substrate is fused silica (FS) or sapphire (Sap), since the densities of these materials differ by a factor of about 2.)

As shown in figure 5, for the sequences of beam widths considered in section 4.1, $w$ and $D$ can approach or even exceed $H$ while simultaneously being significant fractions of the $R$. Consequently, edge effects (finite test-mass effects) may significantly change the noise scalings depicted in figures 3 and 4.

To estimate the importance of these edge effects, I compare the results in sections 4.1.14.1.3 to the finite-test-mass (FTM) results ${ }^{7}$ of Agresti and DeSalvo [10] (all types of thermal

7 The FTM data used here assume that the coating extends all the way to the edge of the substrate face. In advanced LIGO, the coating radius will actually be several $\mathrm{mm}$ smaller than the substrate radius (the baseline substrate radius for advanced LIGO is $170 \mathrm{~mm}$ ). 

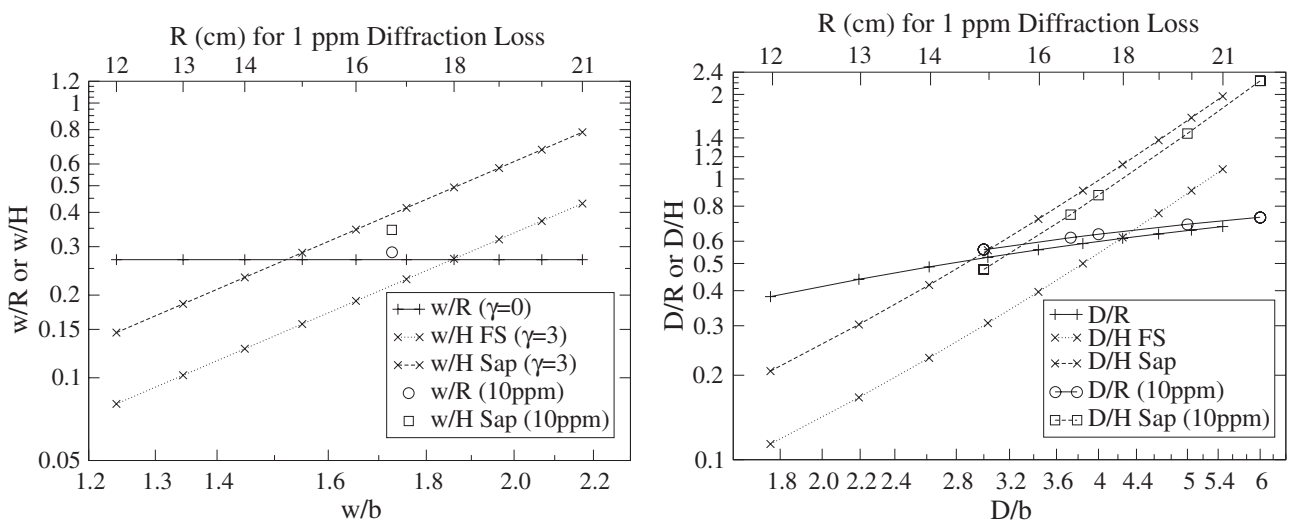

Figure 5. How the Gaussian beam width parameter $w$ (left panel) and mesa beam width parameter $D$ (right panel) compare to the mirror radius $R$ and thickness $H$, when (i) the radius $R$ is fixed so the clipping-approximation diffraction loss is $1 \mathrm{ppm}$ (unless a $10 \mathrm{ppm}$ loss is indicated), and (ii) the thickness $H$ is then determined by holding the mass at $40 \mathrm{~kg}$, the advanced-LIGO baseline mirror mass. The mirror radius $R$ for $1 \mathrm{ppm}$ losses is shown on the top axis; the $10 \mathrm{ppm}$ mirror radii are $R^{10 \mathrm{ppm}}=15.7 \mathrm{~cm}$ (left panel) and $R^{10 \mathrm{ppm}}=13.94 \mathrm{~cm}, 15.7 \mathrm{~cm}, 16.37 \mathrm{~cm}, 18.85 \mathrm{~cm}$, and $21.36 \mathrm{~cm}$ (right panel, from left to right). FS and Sap mean fused-silica and sapphire substrates. Each curve in the left panel is proportional to $w^{\gamma}$.

noise, 1 ppm CADL) and O'Shaughnessy, Strigin and Vyatchanin [8] (substrate thermoelastic noise only, $10 \mathrm{ppm}$ CADL). Specifically, from these data I read off the ratio

$$
C_{\mathrm{FTM}}\left[\tau ; p_{u}(r), p_{k}(r)\right] \equiv \sqrt{\frac{S_{\tau, u}^{\mathrm{FTM}}}{S_{\tau, k}^{\mathrm{FTM}}}}
$$

This change in noise can be compared to $C_{\text {ITM }}\left[\tau ; p_{k}(r), p_{u}(r)\right]$, the change in noise obtained by the ITM approximation. Specifically, if

$$
\Delta\left[\tau ; p_{u}(r), p_{k}(r)\right] \equiv \frac{C_{\mathrm{FTM}}\left[\tau ; p_{u}(r), p_{k}(r)\right]}{C_{\mathrm{ITM}}\left[\tau ; p_{u}(r), p_{k}(r)\right]},
$$

then $|1-\Delta|$ is the fractional error made by using the ITM approximation to compute $C\left[\tau ; p_{u}(r), p_{k}(r)\right]$.

In the following subsections, I consider the errors $|1-\Delta|$ made (sections 4.1.1-4.1.3) by neglecting FTM effects.

4.2.1. Resized Gaussian beam. Figure 6 plots $\Delta\left[\tau ; p_{\text {gauss }}(w ; r), p_{\text {gauss }}\left(w_{o} ; r\right)\right]$ for mirror substrates made of fused silica, the baseline material for advanced LIGO mirrors [1]. For comparison, the figure also shows the corresponding values of $\Delta$ for sapphire substrates.

When the substrate is fused silica, the ITM and FTM scaling laws agree to better than about $10 \%$ so long as $R \lesssim 17 \mathrm{~cm}$, the advanced-LIGO baseline mirror radius [1]. As $R$ increases beyond about $17 \mathrm{~cm},|1-\Delta|$ increases dramatically (to about $50 \%$ for fused silica when $R=21 \mathrm{~cm}$ ), because for such large radii the noise increases (e.g. [8, 10]) with $R$, while the ITM scaling laws predict (figure 3 ) that the noise always decreases with increasing $R$.

When the substrate is sapphire, the FTM effects for the thermoelastic noises lead to errors that are comparable to the fused-silica FTM errors. For a mirror radius of ${ }^{8} R=16 \mathrm{~cm}$, the

8 When sapphire was the baseline test-mass material for advanced LIGO (it has since been abandoned in favour of fused silica), the baseline mirror radius was $R=15.7 \mathrm{~cm} \mathrm{[25].}$ 


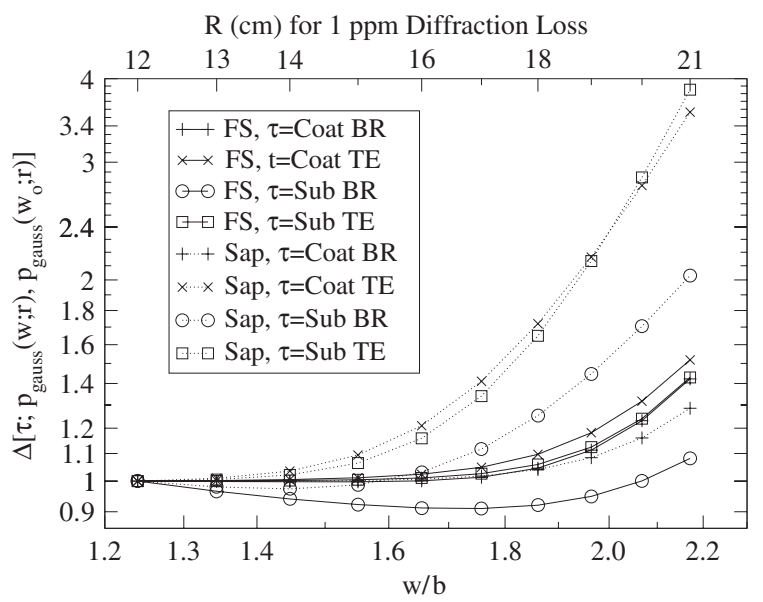

Figure 6. A $\log -\log$ plot of $\Delta\left[\tau ; p_{\text {gauss }}(w ; r), p_{\text {gauss }}\left(w_{o} ; r\right)\right]$. The fractional error of the noise change made by neglecting edge effects is $|1-\Delta|$. Here $w_{o} / b=1.24$, which corresponds to $R=12 \mathrm{~cm}$ and $1 \mathrm{ppm}$ diffraction losses. The FTM values are obtained by taking ratios of the noises calculated by Agresti and DeSalvo [10]. FS and Sap mean fused-silica and sapphire substrates.

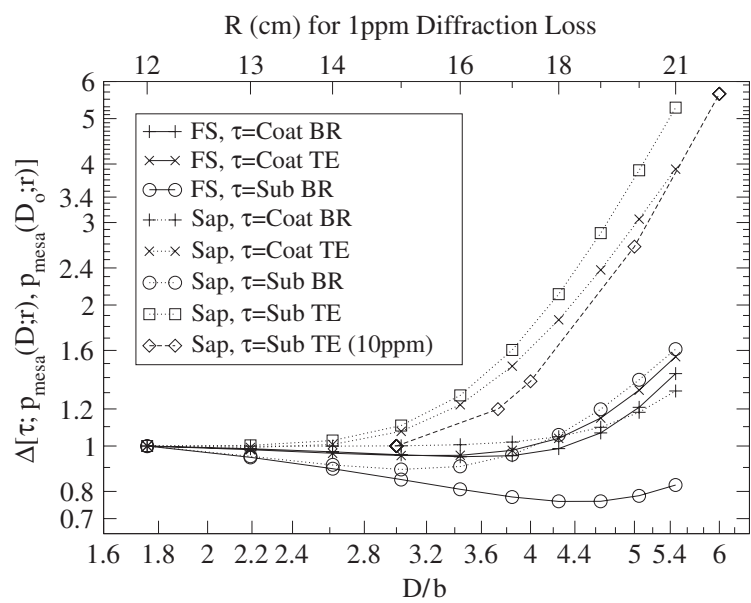

Figure 7. A log-log plot of $\Delta\left[\tau ; p_{\text {mesa }}(D ; r), p_{\text {mesa }}\left(D_{o} ; r\right)\right]$. The fractional error of the noise change made by neglecting edge effects is $|1-\Delta|$. Here the diffraction losses are $1 \mathrm{ppm}$ (unless $10 \mathrm{ppm}$ is indicated), and $D_{o} / b=1.76\left(D_{o}^{10 \mathrm{ppm}}=3.00\right)$, which corresponds to a mirror radius $R=12 \mathrm{~cm}\left(R^{10 \mathrm{ppm}}=13.94 \mathrm{~cm}\right)$. The corresponding mirror radii are given on the top axis ( 1 ppm losses) and in figure 5 (10 ppm losses). The FTM values are obtained by taking ratios of the noises calculated by Agresti and DeSalvo [10], except for the $10 \mathrm{ppm}$ values due to O'Shaughnessy, Strigin and Vyatchanin [8]. FS and Sap mean fused-silica and sapphire substrates. (The fused-silica substrate thermoelastic noise is negligible; this case is omitted from the figure.)

fractional error $|1-\Delta|$ for sapphire substrates is about $15 \%$ for substrate thermoelastic noise and about $20 \%$ for coating thermoelastic noise. 


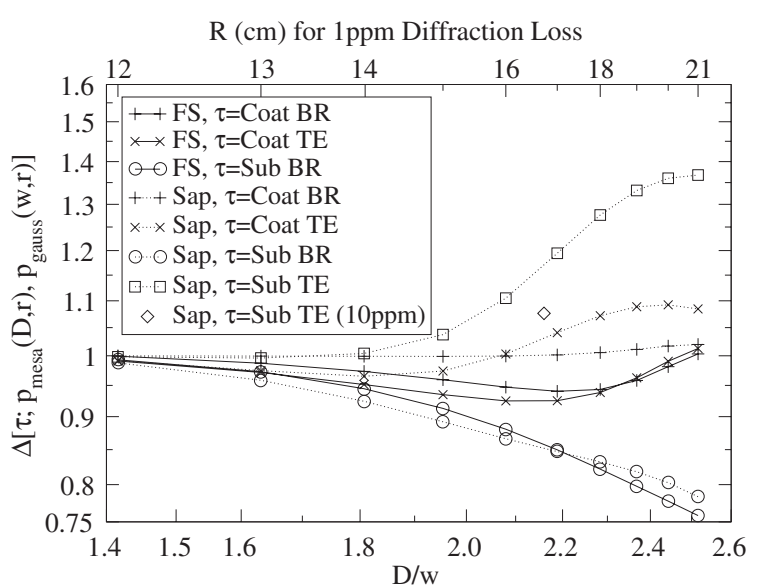

Figure 8. A log-log plot of $\Delta\left[\tau ; p_{\text {mesa }}(D ; r), p_{\text {gauss }}(w ; r)\right]$. The beam width parameters $w$ and $D$ are chosen so that the diffraction loss is $1 \mathrm{ppm}$ (unless $10 \mathrm{ppm}$ is indicated). The corresponding mirror width for $1 \mathrm{ppm}$ diffraction losses is shown on the top axis; the $10 \mathrm{ppm}$ point corresponds to a mirror radius of $15.7 \mathrm{~cm}$. The fractional error of the noise change made by neglecting edge effects is $|1-\Delta|$. The FTM values are obtained by taking ratios of the noises calculated by Agresti and DeSalvo [10], except for the $10 \mathrm{ppm}$ value, which is due to O'Shaughnessy, Strigin and Vyatchanin [8]. FS and Sap mean fused-silica and sapphire substrates. (The fused-silica substrate thermoelastic noise is negligible; this case is omitted from the figure.)

4.2.2. Resized mesa beam. The FTM effects in the resized-mesa-beam case are similar to the resized-Gaussian-beam FTM effects. Figure 7 plots $\Delta\left[\tau ; p_{\text {mesa }}(D ; r), p_{\text {mesa }}\left(D_{o} ; r\right)\right]$. When the substrate is fused silica and $R \lesssim 17 \mathrm{~cm}$, the ITM scaling law errs by less than about $10 \%$ for the coating noises and by less than about $25 \%$ for the substrate Brownian noise. (The substrate thermoelastic noise is negligible when the substrate is fused silica [10].) Again, the ITM scaling law disagrees more and more strongly as $R$ is increased beyond $17 \mathrm{~cm}$. In this regime, the noise increases with $R$, but the ITM scaling law (figure 3) predicts that the noise always decreases with increasing $R$.

When the substrate is sapphire, the FTM effects for the thermoelastic noises are comparable to the Brownian-substrate errors for fused silica. When $R=16 \mathrm{~cm}$, the FTM effects on the sapphire thermoelastic noises correspond to a fractional error $|1-\Delta|$ of $20-30 \%$.

4.2.3. Switching from a Gaussian beam to a mesa beam with the same diffraction loss and mirror radius. The errors due to neglecting FTM effects in the Gaussian-to-mesa case behave qualitatively differently from (and are generally smaller than) the resized-beam errors. Figure 8 plots $\Delta\left[\tau ; p_{\text {mesa }}(D ; r), p_{\text {gauss }}(w ; r)\right]$ for fused silica and sapphire substrates. For both fused-silica and sapphire substrates, the coating noise changes are not strongly sensitive to edge effects; in these cases, $C_{\mathrm{FTM}}$ and $C_{\mathrm{ITM}}$ differ by less than about $10 \%$ even when the beam widths exceed $17 \mathrm{~cm}$ (and thus are significant fractions of $R$ and $H$ (cf figure 5)). The substrate noise changes are more sensitive to edge effects, but even then the edge effects remain below about $15 \%$, provided that $R \lesssim 17 \mathrm{~cm}$ for fused-silica substrates and $R \lesssim 16 \mathrm{~cm}$ for sapphire substrates.

\section{Conclusion}

Changing the shape of the laser beam in advanced LIGO can reduce the thermal noise, which is the limiting noise source at frequencies from $40 \mathrm{~Hz}$ to $200 \mathrm{~Hz}$. In the Fourier domain, the 
relations between the thermal noise and the beam shape for semi-infinite mirrors take the form of simple scaling laws. Moreover, the coating thermal noises obey the same local scaling law. These results enable a straightforward comparison of the thermal noises for two different beam shapes when edge effects are neglected. The scaling laws predict the improvement of mesa-beam noises versus Gaussian-beam noises quite well. For $40 \mathrm{~kg}$, fused-silica mirrors, the substrate-noise scaling laws agree with the finite-mirror results within approximately $15 \%$ for mirror sizes no larger than the advanced-LIGO baseline size of about $17 \mathrm{~cm}$; the coatingnoise scaling laws agree with the finite-mirror predictions to better than about $10 \%$. The infinite-test-mass scaling laws are thus a useful tool for estimating optimal beam shapes for advanced LIGO and other future gravitational-wave interferometers: besides estimating how the noise scales with respect to two particular beam shapes, the scaling laws could, in principle, be minimized to find a beam shape that reduces the thermal noise more than the mesa beam does. The latter prospect is currently being investigated by Bondarescu and Chen [13, 14] and by Pierro and collaborators [15].

\section{Acknowledgments}

I would like to thank Kip Thorne for suggesting this problem and for his advice and encouragement. I would also like to thank Juri Agresti for helpful discussions as well as for providing for comparison the data that will be published in [10]. This work was supported in part by NSF grants PHY-0099568 and PHY-0601459. The numerical computations described in this paper were performed using Mathematica version 5.2. The figures, including best fit lines, were prepared using Grace 5.1.18.

\section{Appendix A. Derivation of equations (32) and (19)}

In this appendix, I derive equation (32), which I use in the derivation of the scaling law (37) for Brownian substrate noise. Then, I deduce equation (19), which I use in the derivation of the scaling law (24) for Brownian coating noise.

First, consider the integral

$$
\int_{0}^{\infty} \mathrm{d} r r\left[S_{\varphi \varphi}^{2}-S_{\varphi \varphi}\left(\theta-S_{z z}\right)\right]
$$

Combining equations (14a) and (14e) gives

$$
\theta-S_{z z}=\frac{1}{2 \mu} \int_{0}^{\infty} \mathrm{d} k \mathrm{e}^{-k z} \tilde{p}(k)\left[\frac{-\mu}{\lambda+\mu}+k z\right] k J_{0}(k r) .
$$

Inserting equations (A.2) and (14c) into the left-hand side of equation (A.1) yields

$$
\begin{aligned}
\int_{0}^{\infty} \mathrm{d} r r\left[S_{\varphi \varphi}^{2}\right. & \left.-S_{\varphi \varphi}\left(\theta-S_{z z}\right)\right]=\frac{1}{4 \mu^{2}} \int_{0}^{\infty} \mathrm{d} k \int_{0}^{\infty} \mathrm{d} k^{\prime} \mathrm{e}^{-\left(k+k^{\prime}\right) z} \\
& \times\left[\frac{-\mu}{\lambda+\mu}+k z\right]\left[\frac{-\mu}{\lambda+\mu}+k^{\prime} z\right] \tilde{p}(k) \tilde{p}\left(k^{\prime}\right) I
\end{aligned}
$$

where

$$
I=\int_{0}^{\infty} \mathrm{d} r \frac{J_{1}(k r) J_{1}\left(k^{\prime} r\right)}{r}-k^{\prime} \int_{0}^{\infty} \mathrm{d} r J_{1}(k r) J_{0}\left(k^{\prime} r\right) .
$$

Since $k$ and $k^{\prime}$ are variables of integration, and since aside from $I$ itself, (A.3) is unchanged by letting $k \leftrightarrow k^{\prime}, I$ can be rewritten as

$I=\int_{0}^{\infty} \mathrm{d} r \frac{J_{1}(k r) J_{1}\left(k^{\prime} r\right)}{r}-\frac{1}{2} k^{\prime} \int_{0}^{\infty} \mathrm{d} r J_{1}(k r) J_{0}\left(k^{\prime} r\right)-\frac{1}{2} k \int_{0}^{\infty} \mathrm{d} r J_{1}\left(k^{\prime} r\right) J_{0}(k r)$. 
The integrals in (A.5) are special cases of equations (11.4.33), (11.4.34) and (11.4.42) of [26]:

$$
\begin{aligned}
& \int_{0}^{\infty} \mathrm{d} r \frac{J_{1}(k r) J_{1}\left(k^{\prime} r\right)}{r}=\frac{k^{\prime}}{2 k} \eta\left(k-k^{\prime}\right)+\frac{k}{2 k^{\prime}} \eta\left(k^{\prime}-k\right), \\
& \int_{0}^{\infty} \mathrm{d} r J_{1}(k r) J_{0}\left(k^{\prime} r\right)=\frac{\eta\left(k-k^{\prime}\right)}{k} .
\end{aligned}
$$

Here $\eta$ is the unit step function. Inserting (A.6) and (A.7) into (A.5) shows that

$$
I=0 \Rightarrow \int_{0}^{\infty} \mathrm{d} r r\left[S_{\varphi \varphi}^{2}-S_{\varphi \varphi}\left(\theta-S_{z z}\right)\right]=0
$$

which is equation (32).

Next, combining equations $(17 a)-(17 d)$ shows that

$$
\begin{aligned}
& S_{\varphi \varphi}^{\text {coat }}=\left.S_{\varphi \varphi}\right|_{z=0} \\
& \theta^{\text {coat }}-S_{z z}^{\text {coat }}=\left.\left(\theta-S_{z z}\right)\right|_{z=0} .
\end{aligned}
$$

Thus, setting $z=0$ in (A.8) gives

$$
\int_{0}^{\infty} \mathrm{d} r r\left[\left(S_{\varphi \varphi}^{\mathrm{coat}}\right)^{2}-S_{\varphi \varphi}^{\mathrm{coat}}\left(\theta^{\mathrm{coat}}-S_{z z}^{\mathrm{coat}}\right)\right]=0
$$

which is equation (19).

\section{Appendix B. Junction conditions for the stress and strain of a statically deformed, semi-infinite mirror with thin coating}

The junction conditions (17a)-(17d) are listed in equation (A4) of [4] along with the statement that for these conditions to hold, the Poisson ratios of the coating and substrate should not be 'too different.' This restriction is actually unnecessary, provided that the coating is sufficiently thin. One can see this as follows:

Because the coating adheres to the substrate surface, the substrate surface and coating have the same tangential displacement. Continuity of $u_{r}$ and $u_{\varphi}$ immediately implies continuity of $S_{r r}$ and $S_{\varphi \varphi}$. (Alternatively, one can argue that $S_{r r}$ and $S_{\varphi \varphi}$ should be continuous across the junction by noting that neither depends on derivatives of $z$.) Also, a straightforward pillbox integration of the equilibrium condition $\nabla_{j} T_{i j}=0$ shows that $T_{z z}$ and $T_{r z}$ are also continuous across the junction.

All of the other junction conditions given in equation (A.4) of [4] then follow, with one exception: the junction condition on $S_{(r z)}$ should read $\mu^{\text {coat }} S_{(r z)}^{\text {coat }}=\mu^{\text {sub }} S_{(r z)}^{\text {sub }}$, not $S_{(r z)}^{\text {coat }}=S_{(r z)}^{\text {sub }}$. But since $T_{r z}=0$ on the coating surface (and thus also to high accuracy throughout the thin coating), this error is irrelevant; the correct junction condition is simply $S_{(r z)}^{\text {coat }}=S_{(r z)}^{\text {sub }}=0$.

\section{References}

[1] Advanced LIGO: context and summary (online document accessed 14 September, 2006) (http://www.ligo. caltech.edu/advLIGO/scripts/summary.shtml)

[2] Agresti J 2005 Researches on non-standard optics for advanced G.W. interferometers (internal LIGO document LIGO-T040225-00-R) (http://www.ligo.caltech.edu/docs/T/T040225-00.pdf)

[3] Liu Y T and Thorne K S 2000 Phys. Rev. D 62122002

[4] Harry G M et al 2002 Class. Quantum Grav. 19897

[5] Braginsky V B and Vyatchanin S P 2003 Phys. Lett. A 312244 
[6] d'Ambrosio E, O'Shaughnessy R and Thorne K S 2000 Beam reshaping to reduce thermal noise (internal LIGO document G000223-00-D) (http://www.ligo.caltech.edu/docs/G/G000223-00.pdf)

[7] D’Ambrosio E et al 2004 Preprint gr-qc/0409075 (submitted to Phys. Rev. D)

[8] O'Shaughnessy R, Strigin S and Vyatchanin S 2003 Preprint gr-qc/0409050 (submitted to Phys. Rev. D)

[9] Agresti J and DeSalvo R 2005 Flat beam profile to depress thermal noise (internal LIGO document G05004100-Z) (http://www.ligo.caltech.edu/docs/G/G050041-00)

[10] Agresti J and DeSalvo R 2007, in preparation

[11] Agresti J et al 2006 J. Phys.: Conf. Ser. 32301

[12] Tarallo M et al 2007 Generation of a flat-top laser beam for gravitational-wave detectors by means of a non-spherical Fabry-Perot resonator Appl. Opt. at press (http://ao.osa.org/upcoming-pdf.cfm?id=80265)

[13] Bondarescu M and Chen Y, in preparation

[14] Bondarescu M 2007 Topics in general relativity PhD Thesis California Institute of Technology (chapter 3) (http://resolver.caltech.edu/CaltechETD:etd-05282007-231321)

[15] Pierro V et al 2007 Perspectives on beam-shaping optimization for thermal-noise reduction in advanced gravitational-wave interferometric detectors: bounds, profiles, and critical parameters (internal LIGO document PO70066-01) (http://www.ligo.caltech.edu/docs/P/P070066-01.pdf) Preprint 0707.0579

[16] Levin Y 1997 Phys. Rev. D 57659

[17] Fejer M M et al 2004 Phys. Rev. D 70082003

[18] O'Shaughnessy R 2006 Preprint gr-qc/0607035v1

[19] O'Shaughnessy R 2006 Class. Quantum Grav. 237627

[20] Vyatchanin S 2004 FDT approach calculations of Brownian noise in thin layer (internal LIGO document T040242-00Z) (http://www.ligo.caltech.edu/docs/T/T040242-00.pdf)

[21] Vinet J Y 2005 Class. Quantum Grav. 221395

[22] Bondu F, Hello P and Vinet J Y 1998 Phys. Lett. A 246227

[23] Siegman A E 1977 Opt. Lett. 113

[24] Advanced LIGO: reference design and astrophysical targets (online document accessed 26 July, 2007) (http://www.ligo.caltech.edu/advLIGO/scripts/ref_des.shtml)

[25] Fritschel P 2001 Advanced LIGO systems design (internal LIGO document t010075-00-d) (http://www.ligo. caltech.edu/docs/T/T010075-00.pdf)

[26] Abramowitz M and Stegun I A (eds) 1964 Handbook of Mathematical Functions (New York: Dover) 